\title{
Minimal residual disease in acute lymphoblastic leukemia: technical aspects and implications for clinical interpretation
}

\author{
In-Suk Kim ${ }^{1,2}$ \\ ${ }^{1}$ Department of Laboratory Medicine, ${ }^{2}$ Research Institute for Convergence of Biomedical Science and Technology, Pusan National \\ University Yangsan Hospital, Yangsan, Korea
}

p-ISSN 2287-979X / e-ISSN 2288-0011 https://doi.org/10.5045/br.2020.S004 Blood Res 2020;55:S19-S26.

Received on November 16, 2019

Revised on January 23, 2020

Accepted on January 23, 2020

\author{
Correspondence to \\ In-Suk Kim, M.D. \\ Department of Laboratory Medicine, \\ Pusan National University Yangsan \\ Hospital, 20 Geumo-ro, Mulgeum-eup, \\ Yangsan 50612, Korea \\ E-mail: iskim0710@gmail.com \\ (C) 2020 Korean Society of Hematology
}

\begin{abstract}
Minimal residual disease (MRD) monitoring has proven to be one of the fundamental independent prognostic factors for patients with acute lymphoblastic leukemia (ALL). Sequential monitoring of MRD using sensitive and specific methods, such as real-time quantitative polymerase chain reaction (qPCR) or flow cytometry (FCM), has improved the assessment of treatment response and is currently used for therapeutic stratification and early detection. Although both FCM and qPCR yield highly consistent results with sensitivities of $10^{-4}$, each method has several limitations. For example, qPCR is time-consuming and laborious: designing primers that correspond to the immunoglobulin (IG) and T-cell receptor (TCR) gene rearrangements at diagnosis can take 3-4 weeks. In addition, the evolution of additional clones beyond the first or index clone during therapy cannot be detected, which might lead to false-negative results. FCM requires experienced technicians and sometimes does not achieve a sensitivity of $10^{-4}$. Accordingly, a next generation sequencing (NGS)-based method has been developed in an attempt to overcome these limitations. With the advent of high-throughput NGS technologies, a more in-depth analysis of $I G$ and/or TCR gene rearrangements is now within reach, which impacts all applications of $I G / T R$ analysis. However, standardization, quality control, and validation of this new technology are warranted prior to its incorporation into routine practice.
\end{abstract}

Key Words Acute lymphoblastic leukemia, Minimal residual diseases, Immunoglobulin, T-cell receptor, Next generation sequencing

\section{INTRODUCTION}

Minimal residual disease (MRD) monitoring has proven to be one of the most substantial independent prognostic factors in patients with acute lymphoblastic leukemia (ALL) $[1,2]$. MRD monitoring is defined as any approach-including cytogenetics, flow cytometry, PCR-based tools, and high throughput sequencing methods-aimed at detecting and possibly quantifying residual tumor cells beyond the sensitivity level of cytomorphology. To be informative, MRD assays for ALL should allow for the detection of 1 leukemic cell among 100,000 normal cells or more in virtually all patients. Currently, standardized methods for studying MRD in ALL are multi-parametric flow cytometry (MFC) of leukemia-associated immunophenotypes (LAIP), and more so, polymerase chain reaction (PCR) amplification-based methods that use leukemia-specific (fusion gene transcripts) or pa- tient-specific [immunoglobulin/T-cell receptor $(I G / T C R)$ gene rearrangements] molecular markers [3-8]. These methods allow for the detection of a single leukemic cell among several normal cells. MRD monitoring based on IG/TCR gene recombinations has been standardized and is widely used $[4,9-12]$.

$I G$ and $T C R$ recombinations occur in the early stages of B-cell and T-cell development. As a consequence, each lymphoid cell contains unique $\mathrm{V}(\mathrm{D}) \mathrm{J}$ recombinations resulting from random coupling between one of several possible $\mathrm{V}$, (D), and J genes, as well as the imprecise joining of gene segments and the addition of nucleotides to the DNA sequence at splice sites. Thus, identical recombinations reflect the clonal nature of a population, rather than being derived from independent cells. Monitoring IG/TCR-based MRD in ALL involves a step-by-step analysis of V(D)J DNA recombinations in lymphoblasts and their subsequent detection, with very high sensitivity, during follow-up. Rapid progression 
in next-generation sequencing (NGS) technologies have enabled highly sensitive cancer genomic testing in clinical laboratories, and NGS-based clonal $I G$ and $T C R$ rearrangement assays have already been tried for MRD monitoring of ALL [13-22]. In this review, we describe various tests to detect MRD, with an emphasis on the importance of developing sensitive systems for improving diagnostic workups and MRD assessments.

\section{METHODOLOGIES FOR MRD DETECTION}

\section{Source of material for MRD evaluation}

In the past, it has been debated if peripheral blood (PB), rather than bone marrow (BM), could be used for MRD monitoring, regardless of the technique used (MFC or PCR). In fact, in B-ALL, MRD levels tend to be 1-3 logs lower in $\mathrm{PB}$ than in $\mathrm{BM}[23,24]$, and blood analyses cannot replace bone marrow assessments. T-lineage ALL MRD assessments are typically carried out on BM samples.

\section{Timepoint of MRD assessment}

MRD is a time point-dependent variable. MRD levels at different time points have different prognostic values for relapse: early MRD assessment identifies patients with a rapid tumor clearance and a very low risk of relapse, whereas any persisting $\mathrm{MRD}$ at the end of consolidation therapy is associated with a particularly poor prognosis.

\section{Multi-parametric flow cytometry analysis}

This approach takes advantage of the presence of protein antigens in the nucleus, cytoplasm, or surface of the cell, which are sequentially acquired during normal cell development. The LAIP must be identified at diagnosis, before any therapy is carried out for each ALL case, by comparing the marker profile of leukemia cells to reference bone marrow samples. Marker profile comparisons are achieved through various combinations of monoclonal antibodies against surface, cytoplasmic, or nuclear leukocyte antigens. A second approach is represented by the so-called "different from normal (DFN)" analysis, which identifies leukemic blasts by recognizing immunophenotypic changes with respect to a normal counterpart population (either hematopoietic progenitors of similar lineage and maturational stage) through the evaluation of antigenic pattern expressions $[25,26]$. At present, the most commonly used MFC panels comprise 6-8 monoclonal antibody (MoAb) combinations. The refinement of MFC has required a parallel advancement in hardware, software, and reagents.

Flow cytometry can be successfully applied to the majority of cases $(>90 \%)$ and can reach a sensitivity of $10^{-4}$. Flow cytometry analysis is quick, and can produce MRD evaluations in only a few hours, and is, therefore, also useful to assess the therapeutic response following the first 2 induction weeks [27, 28]. However, some limitations do exist, such as the fact that samples must be analyzed immediately after collection to avoid cell death, a problem that arises when shipment is required for the centralized evaluation of MRD referral laboratories. Furthermore, a) post-induction regeneration of normal lymphoid cells co-expressing some ALL-type antigens can lead to false-positive results in B-ALL cases, b) the bone marrow sample hypocellularity and, in some patients, a phenotypic shift can induce erroneous or difficult interpretations [29]. The EuroFlow Consortium has optimized and standardized immunostaining protocols for the diagnosis, classification, and prognostic subclassification of hematologic malignancies as well as for the detection of MRD during the clinical follow-up. However, experienced operators are still needed to correctly evaluate MRD results $[30,31]$.

\section{Molecular analysis with antigen-receptor gene rearrangements}

The most commonly used technique is the molecular study-based approach of antigen-receptor gene rearrangements, i.e., IG/TCR gene rearrangements. The study of $I G / T C R$ gene rearrangements has become the most sensitive method to assess the clonality of a lymphoid expansion. Although $I G$ rearrangements are mostly found in B-cells, and $T C R$ rearrangements in T- lymphocytes, both B-lineage and T-lineage leukemic cells can display cross-lineage rearrangements, which can be used for MRD evaluation [9, 32]. To identify these molecular markers at diagnosis, genomic DNA derived from leukemic cells need to undergo a PCR amplification process, where positive PCR products are then analyzed by heteroduplex or gene scan [33] to establish clonality. Subsequently, clonal PCR fragments undergo Sanger sequencing to define the junctional regions and to obtain complementary allele-specific oligonucleotide (ASO)-primers for MRD monitoring, which is typically performed by real-time quantitative PCR (qPCR). Amplification conditions and sensitivity testing for each ASO primer is established by serially diluting the diagnostic material in normal mononuclear cells. This qPCR protocol combined with fluorescently labeled probes allows for the detection of up to 1 leukemic cell in $100,000\left(10^{-5}\right)$ normal lymphoid cells, and leukemia cell dilutions are therefore used to quantify $\mathrm{MRD}$ in bone marrow samples collected during treatment [34]. This technology can generate at least 1 single sensitive molecular probe suitable for MRD analysis in over $90 \%$ of pediatric [4] and adult [2, 35] ALL patients. IG/TCR gene rearrangement analysis is the most widely applied method for MRD detection and has been extensively standardized by the EuroMRD Consortium (previously known as European Study Group- ESG-MRD-ALL). Furthermore, the EuroMRD Consortium is responsible for establishing guidelines for the analysis and interpretation of qPCR data [36, 37], allowing for a favorable homogeneous application of MRD studies within different treatment protocols for childhood and adult ALL. Overall, it is not possible to perform qPCR-based MRD assessments in approximately $5-10 \%$ of ALL cases. Another limitation of this approach is represented by the clonal evolution of $I G / T C R$ rearrangement patterns during the course of the disease and at relapse, which can 
sometimes occur in cases with oligoclonal rearrangements leading to false-negative MRD results [38]. These events depend on the type of marker, disease, and time to relapse (i.e., early or late relapse). The amount of diagnostic DNA is another problematic factor for this type of MRD assessment because diagnostic DNA is needed for each MRD experiment, as well as for the detection of $I G / T C R$ clonal rearrangements, which quantifies the relationship to the tumor load at diagnosis. Finally, qPCR is not able to precisely define the amount of residual disease in cases where the disease burden is very low. Cases with low MRD levels are defined as "positive-not-quantifiable" (PNQ), and their identification currently represents a primary unmet need for clinical utility when treatment decisions are based on MRD monitoring.

\section{Molecular analysis with fusion transcripts}

Another method for molecular MRD detection and monitoring is based on fusion transcript analysis. Overall, more than $40 \%$ of ALL patients carry chromosomal translocations that generate chimeric transcripts. These are potentially ideal targets for MRD assessment [39], since they are critical primary events that are expressed in all leukemic cells, and are incredibly stable during the course of the disease. Within B-lineage ALL, the most common translocation detected in adult cases is the Philadelphia translocation $(\mathrm{Ph})$, also called the Philadelphia chromosome, leading to the breakpoint cluster region-Abelson murine leukemia viral oncogene homolog 1 ( $B C R-A B L 1)$ rearrangement ( $25-30 \%$ of cases). The frequency of the $B C R-A B L 1$ rearrangement increases with age, being detected in approximately $50 \%$ of cases in those above the age of 50 years. At variance, the most common chimeric transcript in pediatric patients is represented by ETS variant transcription factor 6-Runt-related transcription factor 1 (ETV6-RUNX1), which accounts for $25-30 \%$ of childhood ALL. Other fusion transcripts are Lysine methyltransferase 2A-AF4/FMR2 family member 1 (KMT2A-AFF1) and Transcription factor 3-Pre-B-cell leukemia transcription factor 1 ( TCF3-PBXI) each accounting for 3-8\% of cases, regardless of age. Infants (i.e., $<1$ yr) carry a $K M T 2 A$ gene rearrangement in $80 \%$ of cases. In T-ALL, T-cell acute lymphocytic leukemia 1 (TAL1) deletions [SCL interrupting locus (SIL)/TAL1] occur in about $20 \%$ of T-ALL. Other rarer translocations in T-ALL involve the $A B L 1$ gene; for example, Nucleoporin (NUP)/ABL1, Echinoderm microtubule-associated protein-like 1 (EML1)/ABL1, and ETVG/ABL1. Due to the majority of these chromosomal abnormalities have a prognostic value, their detection must be performed in all cases at diagnosis $[8,40-43]$ so that each patient can be monitored for MRD using a predefined marker throughout the course of the disease. Ribonucleic acid (RNA) is the optimal starting material to detect these lesions, allowing for the use of a small number of quantitative reverse transcriptase-PCR (RT-PCR) assays. This offers the opportunity to apply the same primer set to all patients bearing the same translocation, leading to an easy and rapid fusion transcript evaluation at diagnosis and during treatment [39]. Quantification of the fusion gene using RNA samples is achieved by comparing the amplified product to a standard curve derived from the amplification of serial dilutions of a cell line or plasmid DNA (i.e., $B C R-A B L 1+$ ). This highly sensitive MRD assay is capable of detecting up to 1 leukemic cell within 100,000 $\left(10^{-5}\right)$ normal lymphoid cells. Moreover, it is not patient-specific, is relatively easy to perform, and is not expensive.

\section{Next-generation flow cytometry}

The novel next generation flow (NGF)-MRD approach takes advantage of innovative tools and procedures recently developed by the EuroFlow Consortium for sample preparation, antibody combinations (including choice of type of antibody and fluorochrome), and identification of B-ALL pathways in the BM, which allows to define the degree of immunophenotypic deviation of B-ALL cells from normal $\mathrm{B}$-cell precursors (also in regenerating $\mathrm{BM}$ ). Additionally, for T-ALL, a comparable strategy is used to obtain reliable (evidence-based) antibody combinations, in order to discriminate from various types of normal T-cells and other cells with cross-lineage marker expression [44, 45].

NGF-MRD is faster, reproducible, and has greater applicability (>95\%) compared to molecular based MRD detection. Moreover, the costs of reagents and assays are estimated to be lower than those of NGS. NGF-MRD reaches a sensitivity close to $10^{-6}$, while conventional flow tools reach sensitivities in the range of $10^{-4}-10^{-5}$. The higher sensitivity of NGF-MRD is mostly due to the use of standardized approaches, including instrument setting, sample processing with bulk lysis procedure, immunostaining, data acquisition, and data analysis with standardized (even automated) gating strategies for definition of cell populations [46]. However, the acquisition of a large number of cells is needed to reach the required sensitivity. In the forthcoming decade, new flow technologies will improve the applicability and specificity of flow MRD measurements.

\section{Digital-droplet-PCR}

The digital PCR technology (ddPCR) has the potential to overcome the limitations of qPCR. DdPCR is the third-generation implementation of conventional PCR that allows the quantification of nucleic acid targets without the need of calibration curves [47, 48]. As reported in several studies $[49,50]$, based on the dynamic nature of the two methods, ddPCR appears to be more accurate than qPCR since: i) each sample is partitioned in droplets and each droplet is analyzed individually, ii) small changes in fluorescence intensity are more readily detected, and iii) the ratio between target DNA molecules to PCR reagents is substantially higher; his increases its amplification efficiency. Finally, the presence of inhibitors negatively affects qPCR efficiency but not that of ddPCR.

The ddPCR technology has been applied to various fields of medical diagnostics, in particular in molecular oncology [47-51], in prenatal diagnosis, and in hematologic malignancies $[47,52-55]$ that are available in the literature. These studies have established analytical parameters to investigate the applicability of ddPCR for MRD detection and concluded 
that ddPCR has a sensitivity, accuracy, and reproducibility at least comparable to that of qPCR. Regarding MRD evaluations, some discordances have been observed at very low disease levels; in this setting, ddPCR showed an excellent analytical performance to quantify those low positive samples defined as PNQ by qPCR or to identify the false MRD+ cases. Recently, the clinical significance of ddPCR has been reported in a pediatric cohort of ALL patients [21]. Nevertheless, no established guidelines for ddPCR MRD analysis and interpretation have so far been defined. A major standardization effort is underway within the EuroMRD Consortium.

\section{Next generation sequencing}

Several groups have shown the value of NGS technologies for MRD detection in precursor and mature B-cell tumors [13-18, 56-58]. NGS can be used to detect clone-specific $I G / T C R$ index sequences; clonal sequences detected at diagnosis can be re-detected and quantified in each follow-up sample. By using universal primers, this method allows for monitoring all IG/TCR gene rearrangements concertedly, providing a complete picture not only of the residual leukemia but also of the normal immune repertoire [17].

Sensitivity is a critical aspect of MRD detection. Methods allowing a sensitivity higher than $10^{-5}$ (routinely achieved by qPCR) might be of interest to identify very low-level diseases. Studies using the NGS platform in ALL and chronic lymphocytic leukemia have demonstrated that a sensitivity level of $10^{-6}$ [59], is achievable when higher amounts of DNA are used. This is reflected in the possibility of detecting early clonal evolution, a relatively frequent occurrence in relapsed ALL [60].

Many authors have reported that NGS appears to be more specific than qPCR in predicting relapse in ALL patients after induction [17] as well as after allogenic SCT [61]. A comparative MRD analysis between qPCR and NGS showed within the Berlin Frankfurt Münster (BFM) trials a change in the stratification risk, mainly due to different interpretations of the two techniques within low-positive samples [62]. The NGS quantitative discrimination is always superimposable to the sensitivity, whilst the qPCR quantitative range is usually inferior to the sensitivity threshold leading to cases which are defined as PNQ as previously described [59].

However, NGS has a substantial intrinsic complexity and involves major costs. Furthermore, current robust and broadly applicable NGS-based MRD standardized protocols are still not available in academic laboratories. The EuroclonalityNGS Consortium is working to standardize guidelines for analysis and data interpretation. DdPCR and NGS appear to be feasible and attractive alternative methods for MRD assessment that can help to more precisely classify cases that qPCR is unable to detect or quantify.

\section{CLINICAL SIGNIFICANCE OF MRD}

\section{Correct interpretation of MRD results}

Like other quantitative methods, MRD quantification techniques have a lower limit of detection and a lower limit of quantification. Therefore, MRD negativity is not synonymous with the absence of residual disease, which is why several authors use the term "measurable residual disease" instead of "minimal residual disease." The sensitivity of measurements is determined by the particular technique, and the amount of cell correlates analyzed. Current treatment protocols require a sensitivity of at least $10^{-4}$. Some recent studies have shown that the use of commercial approaches for NGS MRD detection claim to reach sensitivities down to $10^{-7}$ [63]. However, it is important to note that the amount of input DNA is crucial for reaching a particular sensitivity. This often represents a serious limitation in the aplastic samples during treatment [1]. An MRD assessment using 100,000 cells can never reach a sensitivity of $10^{-6}$, even if the readout suggests that it did. It seems that IG/TCR and $B C R-A B L 1$ MRD may provide distinct insights into MRD kinetics of different leukemic subpopulations in response to tyrosine kinase inhibitors, chemotherapy, SCT, and possibly immunotherapies.

To allow for a correct interpretation of MRD results, the MRD report must provide information on the MRD technique used, MRD markers, theoretical limit of detection, and limit of quantification of the assay.

\section{MRD in the setting of chemotherapy}

The clinical impact of MRD is now widely accepted and is regarded today as the most important prognostic factor in the state-of-the-art management of ALL. MRD can provide different information, according to the timing in which it is performed (very early during treatment, after induction/ consolidation, and before and after SCT) and, more recently, it can be refined by the evaluation of additional genomic markers.

The initial MRD response to therapy is a relevant prognostic factor in both childhood and adult ALL [2]; indeed, MRD negativity at very early time points during induction therapy correlates with a particularly excellent outcome both in childhood and adult ALL, and is indicative of a high sensitivity to chemotherapy. The first pivotal study on molecular MRD analysis was carried out by the German Multicenter Study Group for Adult ALL (GMALL) on a large cohort of $\mathrm{Ph}$-patients with standard-risk and high-risk features $(\mathrm{N}=580)$ that showed that the molecular response to standard induction and consolidation treatment was the only significant prognostic factor for remission duration and survival in both risk groups [64]. These data have been confirmed by other groups, regardless of the cut-off values, MRD technique, the timing of MRD analysis, and the target patient population chosen. 
MRD and stem cell transplantation

As mentioned, MRD after induction/early consolidation is the most critical decision-making parameter for an allogeneic transplant. A recent meta-analysis on 21 published reports, including over 2,000 patients, confirmed that a pre-transplant positive MRD is a significant negative predictor of relapse-free survival (RFS), event-free survival (EFS), and OS; as expected, a positive MRD prior to transplant was not associated with a higher rate of non-relapse mortality [65]. Therefore, these results show that MRD evaluation before transplant is extremely useful for treatment intensification since we can offer the opportunity to adequately use immunotherapeutic compounds (e.g., blinatumomab, inotuzumab, and in the future possibly CAR-T cells) aimed at obtaining an MRD negative status.

With regard to the post-transplant setting, MRD monitoring has been much less frequently used after SCT, primarily due to that donor chimerism monitoring provides an alternative for early relapse detection. Nevertheless, it has been shown that an IG/TCR-based MRD assessment allowed for earlier and more specific detection of an impending relapse compared to chimerism analysis, showing that MRD positivity was an independently significant predictor of the risk of relapse [66].

\section{Relapse and clonal evolution}

At relapse, molecular evaluation of $I G / T C R$ markers previously used for $\mathrm{MRD}$ monitoring might be useful to confirm the persistence of the same clone. However, it has some potential pitfalls, mostly represented by the phenomenon of clonal evolution. The analysis of all molecular markers at first diagnosis and at the time of relapse may reveal a different origin of the predominant clone [60]. Indeed, the loss of a molecular marker at relapse, or the expansion of a clonal marker expressed at the subclonal level at diagnosis, is a relatively frequent event. Therefore, the MRD assessment should be carried out with several targets [60]. Finally, it is worth being mindful that the clonal evolution can vary according to the site of relapse (medullary or extramedullary).

\section{MRD and novel agents}

Novel therapies, such as monoclonal antibodies, bispecific $\mathrm{T}$-cell engagers, or chimeric antigen receptor T-cells (CART), are an exciting advancement in the immunotherapeutic treatment of relapse/refractory B-ALL. These new therapeutic approaches make MRD an almost perfect therapeutic target, considering that MRD+ patients harbor significantly less leukemic cells and, therefore, a more manageable clinical profile than cases in hematologic relapse.

\section{CONCLUSION}

MRD is a powerful and independent predictor of outcomes in both children and adult ALL, during treatment, in the pre- and post-SCT settings, with different prognostic meanings based on the clinical context. However, MRD is a con- text-dependent variable with different prognostic meanings in first-line treatment compared with salvage therapy, $\mathrm{Ph}-$ compared with $\mathrm{Ph}+-\mathrm{ALL}$, and for early response assessment against post-remission monitoring. Molecular rearrangements (gene fusion and $I G / \mathrm{TR}$ gene rearrangements) are widely used as targets to detect residual leukemic cells in ALL patients, although new molecular markers could be used for prognostic and therapeutic purposes to improve the number of evaluable patients. Technically, MFC and qPCR are the most broadly applied consolidated methods for MRD monitoring, although they present intrinsic limitations that must necessarily be overcome. DdPCR, NGS, and NGF, the standardization of which is underway, have shown to be important tools to evaluate MRD in the research setting, and will most likely soon be incorporated in clinical trials due to their ability to overcome the limitation of standard approaches. Therefore, precise MRD cutoff levels, techniques used, and optimal sampling time points must be defined for each treatment protocol before MRD-based treatment stratification can be implemented.

\section{Authors' Disclosures of Potential Conflicts of Interest}

No potential conflicts of interest relevant to this article were reported.

\section{REFERENCES}

1. van Dongen JJ, van der Velden VH, Brüggemann M, Orfao A. Minimal residual disease diagnostics in acute lymphoblastic leukemia: need for sensitive, fast, and standardized technologies. Blood 2015;125:3996-4009.

2. Brüggemann M, Gökbuget N, Kneba M. Acute lymphoblastic leukemia: monitoring minimal residual disease as a therapeutic principle. Semin Oncol 2012;39:47-57.

3. van der Velden VH, Joosten SA, Willemse MJ, et al. Real-time quantitative PCR for detection of minimal residual disease before allogeneic stem cell transplantation predicts outcome in children with acute lymphoblastic leukemia. Leukemia 2001;15:1485-7.

4. Flohr T, Schrauder A, Cazzaniga G, et al. Minimal residual disease-directed risk stratification using real-time quantitative PCR analysis of immunoglobulin and T-cell receptor gene rearrangements in the international multicenter trial AIEOPBFM ALL 2000 for childhood acute lymphoblastic leukemia. Leukemia 2008;22:771-82.

5. van der Velden VH, van Dongen JJ. MRD detection in acute lymphoblastic leukemia patients using Ig/TCR gene rearrangements as targets for real-time quantitative PCR. Methods Mol Biol 2009;538:115-50.

6. Campana D. Molecular determinants of treatment response in acute lymphoblastic leukemia. Hematology Am Soc Hematol Educ Program 2008;2008:366-73.

7. Dworzak MN, Gaipa G, Ratei R, et al. Standardization of flow cytometric minimal residual disease evaluation in acute lymphoblastic leukemia: Multicentric assessment is feasible. 
Cytometry B Clin Cytom 2008;74:331-40.

8. Elia L, Grammatico S, Paoloni F, et al. Clinical outcome and monitoring of minimal residual disease in patients with acute lymphoblastic leukemia expressing the MLL/ENL fusion gene. Am J Hematol 2011;86:993-7.

9. van Dongen JJ, Langerak AW, Brüggemann M, et al. Design and standardization of PCR primers and protocols for detection of clonal immunoglobulin and $\mathrm{T}$-cell receptor gene recombinations in suspect lymphoproliferations: report of the BIOMED-2 Concerted Action BMH4-CT98-3936. Leukemia 2003;17:2257317.

10. Kreyenberg H, Eckert C, Yarkin Y, et al. Immunoglobulin and $\mathrm{T}$-cell receptor gene rearrangements as PCR-based targets are stable markers for monitoring minimal residual disease in acute lymphoblastic leukemia after stem cell transplantation. Leukemia 2009;23:1355-8.

11. Donovan JW, Ladetto M, Zou G, et al. Immunoglobulin heavy-chain consensus probes for real-time PCR quantification of residual disease in acute lymphoblastic leukemia. Blood 2000;95:2651-8.

12. Jonsson OG, Kitchens RL, Scott FC, Smith RG. Detection of minimal residual disease in acute lymphoblastic leukemia using immunoglobulin hypervariable region specific oligonucleotide probes. Blood 1990;76:2072-9.

13. Theunissen PMJ, de Bie M, van Zessen D, de Haas V, Stubbs AP, van der Velden VHJ. Next-generation antigen receptor sequencing of paired diagnosis and relapse samples of B-cell acute lymphoblastic leukemia: clonal evolution and implications for minimal residual disease target selection. Leuk Res 2019;76: 98-104.

14. Shin S, Hwang IS, Kim J, Lee KA, Lee ST, Choi JR. Detection of immunoglobulin heavy chain gene clonality by next-generation sequencing for minimal residual disease monitoring in B-lymphoblastic leukemia. Ann Lab Med 2017;37:331-5.

15. Sala Torra O, Othus M, Williamson DW, et al. Next-generation sequencing in adult $\mathrm{B}$ cell acute lymphoblastic leukemia patients. Biol Blood Marrow Transplant 2017;23:691-6.

16. Reyes-Barron C, Burack WR, Rothberg PG, Ding Y. Nextgeneration sequencing for minimal residual disease surveillance in acute lymphoblastic leukemia: an update. Crit Rev Oncog 2017;22:559-67.

17. Kotrova M, Trka J, Kneba M, Brüggemann M. Is next-generation sequencing the way to go for residual disease monitoring in acute lymphoblastic leukemia? Mol Diagn Ther 2017;21:481-92.

18. Inaba $\mathrm{H}$, Azzato EM, Mullighan CG. Integration of nextgeneration sequencing to treat acute lymphoblastic leukemia with targetable lesions: The St. Jude Children's Research Hospital Approach. Front Pediatr 2017;5:258.

19. Heikamp EB, Pui CH. Next-generation evaluation and treatment of pediatric acute lymphoblastic leukemia. J Pediatr 2018;203: 14-24.e2.

20. Germano G, Valsecchi MG, Buldini B, et al. Next-generation sequencing of PTEN mutations for monitoring minimal residual disease in T-cell acute lymphoblastic leukemia. Pediatr Blood Cancer 2020;67:e28025.

21. Della Starza I, De Novi LA, Santoro A, et al. Digital droplet PCR and next-generation sequencing refine minimal residual disease monitoring in acute lymphoblastic leukemia. Leuk Lymphoma 2019;60:2838-40.

22. Coccaro N, Anelli L, Zagaria A, Specchia G, Albano F. Nextgeneration sequencing in acute lymphoblastic leukemia. Int J Mol Sci 2019;20:E2929.

23. Coustan-Smith E, Sancho J, Hancock ML, et al. Use of peripheral blood instead of bone marrow to monitor residual disease in children with acute lymphoblastic leukemia. Blood 2002;100: 2399-402.

24. Theunissen P, Mejstrikova E, Sedek L, et al. Standardized flow cytometry for highly sensitive MRD measurements in B-cell acute lymphoblastic leukemia. Blood 2017;129:347-57.

25. Chen X, Wood BL. Monitoring minimal residual disease in acute leukemia: Technical challenges and interpretive complexities. Blood Rev 2017;31:63-75.

26. Sarmiento Palao H, Tarín F, Martirena F, et al. A reproducible strategy for analysis of minimal residual disease measured by Standardized multiparametric flow cytometry in b acute lymphoblastic leukemia. Cytometry B Clin Cytom 2019;96:12-5.

27. Basso G, Veltroni M, Valsecchi MG, et al. Risk of relapse of childhood acute lymphoblastic leukemia is predicted by flow cytometric measurement of residual disease on day 15 bone marrow. J Clin Oncol 2009;27:5168-74.

28. Ratei R, Basso G, Dworzak M, et al. Monitoring treatment response of childhood precursor B-cell acute lymphoblastic leukemia in the AIEOP-BFM-ALL 2000 protocol with multiparameter flow cytometry: predictive impact of early blast reduction on the remission status after induction. Leukemia 2009;23:528-34.

29. Dworzak MN, Gaipa G, Schumich A, et al. Modulation of antigen expression in B-cell precursor acute lymphoblastic leukemia during induction therapy is partly transient: evidence for a drug-induced regulatory phenomenon. Results of the AIEOPBFM-ALL-FLOW-MRD-Study Group. Cytometry B Clin Cytom 2010;78:147-53.

30. Kalina T, Flores-Montero J, van der Velden VH, et al. EuroFlow standardization of flow cytometer instrument settings and immunophenotyping protocols. Leukemia 2012;26:1986-2010.

31. Kalina T, Brdickova N, Glier H, et al. Frequent issues and lessons learned from EuroFlow QA. J Immunol Methods 2018;475: 112520.

32. Szczepański T, Beishuizen A, Pongers-Willemse MJ, et al. Cross-lineage $\mathrm{T}$ cell receptor gene rearrangements occur in more than ninety percent of childhood precursor-B acute lymphoblastic leukemias: alternative PCR targets for detection of minimal residual disease. Leukemia 1999;13:196-205.

33. Germano G, Songia S, Biondi A, Basso G. Rapid detection of clonality in patients with acute lymphoblastic leukemia. Haematologica 2001;86:382-5.

34. Verhagen OJ, Willemse MJ, Breunis WB, et al. Application of germline IGH probes in real-time quantitative PCR for the detection of minimal residual disease in acute lymphoblastic leukemia. Leukemia 2000;14:1426-35.

35. Brüggemann M, Raff T, Flohr $\mathrm{T}$, et al. Clinical significance of minimal residual disease quantification in adult patients with standard-risk acute lymphoblastic leukemia. Blood 2006;107: 1116-23.

36. van der Velden VH, Panzer-Grümayer ER, Cazzaniga G, et al. 
Optimization of PCR-based minimal residual disease diagnostics for childhood acute lymphoblastic leukemia in a multi-center setting. Leukemia 2007;21:706-13.

37. van der Velden VH, Cazzaniga G, Schrauder A, et al. Analysis of minimal residual disease by Ig/TCR gene rearrangements: guidelines for interpretation of real-time quantitative PCR data. Leukemia 2007;21:604-11.

38. Szczepański T, van der Velden VH, Raff T, et al. Comparative analysis of $\mathrm{T}$-cell receptor gene rearrangements at diagnosis and relapse of T-cell acute lymphoblastic leukemia (T-ALL) shows high stability of clonal markers for monitoring of minimal residual disease and reveals the occurrence of second T-ALL. Leukemia 2003;17:2149-56.

39. Gabert J, Beillard E, van der Velden VH, et al. Standardization and quality control studies of 'real-time' quantitative reverse transcriptase polymerase chain reaction of fusion gene transcripts for residual disease detection in leukemia - a Europe Against Cancer program. Leukemia 2003;17:2318-57.

40. Pfeifer H, Cazzaniga G, van der Velden VHJ, et al. Standardisation and consensus guidelines for minimal residual disease assessment in Philadelphia-positive acute lymphoblastic leukemia (Ph + ALL) by real-time quantitative reverse transcriptase PCR of e1a2 BCR-ABL1. Leukemia 2019;33:1910-22.

41. Pfeifer H, Wassmann B, Bethge W, et al. Randomized comparison of prophylactic and minimal residual disease-triggered imatinib after allogeneic stem cell transplantation for BCR-ABL1-positive acute lymphoblastic leukemia. Leukemia 2013;27:1254-62.

42. Hong Y, Zhao X, Qin Y, et al. The prognostic role of E2A-PBX1 expression detected by real-time quantitative reverse transcriptase polymerase chain reaction (RQ-PCR) in B cell acute lymphoblastic leukemia after allogeneic hematopoietic stem cell transplantation. Ann Hematol 2018;97:1547-54.

43. Bolufer P, Barragán E, Verdeguer A, et al. Rapid quantitative detection of TEL-AML1 fusion transcripts in pediatric acute lymphoblastic leukemia by real-time reverse transcription polymerase chain reaction using fluorescently labeled probes. Haematologica 2002;87:23-32.

44. van Dongen JJ, Lhermitte L, Böttcher S, et al. EuroFlow antibody panels for standardized n-dimensional flow cytometric immunophenotyping of normal, reactive and malignant leukocytes. Leukemia 2012;26:1908-75.

45. Pedreira CE, Costa ES, Lecrevisse Q, van Dongen JJ, Orfao A; EuroFlow Consortium. Overview of clinical flow cytometry data analysis: recent advances and future challenges. Trends Biotechnol 2013;31:415-25.

46. Flores-Montero J, Sanoja-Flores L, Paiva B, et al. Next generation flow for highly sensitive and standardized detection of minimal residual disease in multiple myeloma. Leukemia 2017;31: 2094-103.

47. Coccaro N, Anelli L, Zagaria A, et al. Droplet digital PCR is a robust tool for monitoring minimal residual disease in adult Philadelphia-positive acute lymphoblastic leukemia. J Mol Diagn 2018;20:474-82.

48. Sanders R, Huggett JF, Bushell CA, Cowen S, Scott DJ, Foy CA. Evaluation of digital PCR for absolute DNA quantification. Anal Chem 2011;83:6474-84.

49. Whale AS, Huggett JF, Cowen S, et al. Comparison of microfluidic digital PCR and conventional quantitative PCR for measuring copy number variation. Nucleic Acids Res 2012;40:e82.

50. Hindson CM, Chevillet JR, Briggs HA, et al. Absolute quantification by droplet digital PCR versus analog real-time PCR. Nat Methods 2013;10:1003-5.

51. Belgrader P, Tanner SC, Regan JF, Koehler R, Hindson BJ, Brown AS. Droplet digital PCR measurement of HER2 copy number alteration in formalin-fixed paraffin-embedded breast carcinoma tissue. Clin Chem 2013;59:991-4.

52. Lund HL, Hughesman CB, McNeil K, et al. Initial diagnosis of chronic myelogenous leukemia based on quantification of M-BCR status using droplet digital PCR. Anal Bioanal Chem 2016;408: 1079-94.

53. Alikian M, Ellery P, Forbes $M$, et al. Next-generation sequencingassisted dna-based digital PCR for a personalized approach to the detection and quantification of residual disease in chronic myeloid leukemia patients. J Mol Diagn 2016;18:176-89.

54. Zagaria A, Anelli L, Coccaro N, et al. BCR-ABL1 e6a2 transcript in chronic myeloid leukemia: biological features and molecular monitoring by droplet digital PCR. Virchows Arch 2015;467: 357-63.

55. Jennings LJ, George D, Czech J, Yu M, Joseph L. Detection and quantification of BCR-ABL1 fusion transcripts by droplet digital PCR. J Mol Diagn 2014;16:174-9.

56. Wright G, Watt E, Inglott S, Brooks T, Bartram J, Adams SP. Clinical benefit of a high-throughput sequencing approach for minimal residual disease in acute lymphoblastic leukemia. Pediatr Blood Cancer 2019;66:e27787.

57. Salson M, Giraud M, Caillault A, et al. High-throughput sequencing in acute lymphoblastic leukemia: Follow-up of minimal residual disease and emergence of new clones. Leuk Res 2017;53:1-7.

58. Wu J, Jia S, Wang C, et al. Minimal residual disease detection and evolved IGH clones analysis in acute B lymphoblastic leukemia using IGH deep sequencing. Front Immunol 2016;7:403.

59. Ladetto $M$, Brüggemann $M$, Monitillo $L$, et al. Next-generation sequencing and real-time quantitative PCR for minimal residual disease detection in B-cell disorders. Leukemia 2014;28:1299-307.

60. Eckert C, Flohr T, Koehler R, et al. Very early/early relapses of acute lymphoblastic leukemia show unexpected changes of clonal markers and high heterogeneity in response to initial and relapse treatment. Leukemia 2011;25:1305-13.

61. Kotrova M, van der Velden VHJ, van Dongen JJM, et al. Next-generation sequencing indicates false-positive MRD results and better predicts prognosis after SCT in patients with childhood ALL. Bone Marrow Transplant 2017;52:962-8.

62. Kotrova M, Muzikova K, Mejstrikova E, et al. The predictive strength of next-generation sequencing MRD detection for relapse compared with current methods in childhood ALL. Blood 2015;126:1045-7.

63. Faham M, Zheng J, Moorhead M, et al. Deep-sequencing approach for minimal residual disease detection in acute lymphoblastic leukemia. Blood 2012;120:5173-80.

64. Gökbuget N, Kneba M, Raff T, et al. Adult patients with acute lymphoblastic leukemia and molecular failure display a poor prognosis and are candidates for stem cell transplantation and targeted therapies. Blood 2012;120:1868-76. 
65. Shen Z, Gu X, Mao W, et al. Influence of pre-transplant minimal residual disease on prognosis after Allo-SCT for patients with acute lymphoblastic leukemia: systematic review and metaanalysis. BMC Cancer 2018;18:755.

66. Terwey $\mathrm{TH}$, Hemmati PG, Nagy M, et al. Comparison of chimerism and minimal residual disease monitoring for relapse prediction after allogeneic stem cell transplantation for adult acute lymphoblastic leukemia. Biol Blood Marrow Transplant 2014;20:1522-9. 